\title{
Constraints on extremal self-dual CFTs
}

\author{
Matthias R. Gaberdiel* \\ Institut für Theoretische Physik, \\ ETH Zürich \\ CH-8093 Zürich, Switzerland
}

July 2007

\begin{abstract}
We argue that the existence of a modular differential equation implies that a certain vector vanishes in Zhu's $C_{2}$ quotient space, and we check this assertion in numerous examples. If this connection is true in general, it would imply that the recently conjectured extremal self-dual conformal field theories at $c=24 k$ cannot exist for $k \geq 42$.
\end{abstract}

${ }^{*}$ E-mail: gaberdiel@itp.phys.ethz.ch 


\section{Introduction}

Recently Witten has conjectured [1, based on an analysis of pure gravity in $A d S_{3}$, that a family of extremal self-dual meromorphic bosonic conformal field theories at $c=24 k$ (with $k=1,2, \ldots$ ) exists. Here extremal means that, up to level $k+1$ above the vacuum, the theory only contains the Virasoro descendants of the vacuum state. A meromorphic conformal field theory (see [2] for an introduction) is self-dual if its only representation is the vacuum representation itself. In particular, this implies that the vacuum character has to be invariant under the $S$-modular transformation. Taken together, these requirements then determine the vacuum character of this meromorphic conformal field theory uniquely.

For $k=1$ the meromorphic conformal field theory in question is the famous Monster theory [3, 4] (for a beautiful introduction see [5]). But for $k \geq 2$ an explicit realisation of these theories is so far not known. The proposed conformal field theories satisfy however a few consistency conditions. First of all, the coefficients of the $q$-expansion are positive integers, and thus can be interpreted as vacuum characters of conformal field theories. Witten also showed that the $k=2$ vacuum amplitudes are well-defined on higher genus Riemann surfaces [1]; more recently, the genus 2 amplitude of the $k=3$ theory was shown to be consistent (by some other methods) [6]. Their method determines also the genus 2 partition functions uniquely up to $k \leq 10$.

While these are impressive consistency checks, they essentially only test the modular properties of vacuum expectation values, and are thus not very sensitive to the inner workings of the theory. (For example, on the level of the torus amplitudes, one would expect that there are infinitely many self-dual conformal field theories at $c=24$, since one may add to $J(q)=j(q)-744$ any positive integer. On the other hand, it is believed that there are only 71 such theories [7].) It would thus be very desirable to subject these theories to consistency conditions that go beyond these considerations. In this paper we want to make one small step in this direction by analysing the structure of the modular differential equation for these theories.

It has long been known that all the characters of a rational conformal field theory satisfy a common modular differential equation [8, 9]. From a mathematical point of view, this differential equation can be obtained quite generally for theories that satisfy the so-called $C_{2}$ condition [10]; this is believed to be the case for all rational conformal field theories. For theories satisfying this condition, there exists an integer $s$ for which $L_{-2}^{s} \Omega$ plus some correction terms of lower conformal weight lie in a certain subspace $O_{q}\left(\mathcal{H}_{0}\right)$ for precise definitions see section 2. This then leads to a modular differential equation of order s. On the other hand, such a relation in $O_{q}\left(\mathcal{H}_{0}\right)$ can only exist if $L_{-2}^{s} \Omega \in O_{[2]}$. In turn this requires that the vacuum representation possesses a null-vector at conformal weight $2 s$.

It seems very natural to believe (and we shall show that this is at least true in many examples) that every modular differential equation arises in this fashion. Thus if the characters of the chiral algebra satisfy a modular differential equation of order $s$, this suggests that $L_{-2}^{s} \Omega \in O_{[2]}$, which in turn implies that the vacuum representation has to have a null vector at level $2 s$. Applied to the above candidate theories we find that for $k \geq 42$, this predicts the existence of a null vector at a level less than $k+1$. On the other 
hand, since the theory up to level $k+1$ is just the Virasoro theory at $c=24 k$, we know that no such null vector exists. Thus our analysis suggests that at least the theories with $k \geq 42$ are inconsistent.

The paper is organised as follows. In the next section we review the modular differential equation from the point of view of [10]. In section 3 we explain why we expect the order of the modular differential equation to be related to the property that $L_{-2}^{s} \Omega \in O_{[2]}$. We also check this claim explicitly for a number of theories, in particular, the minimal models, the $\mathrm{su}(2)$ affine theories, $\mathrm{su}(3)$ at level $k=1,2$ and the self-dual theories corresponding to $e_{8}$ (at level $k=1$ ), $e_{8} \oplus e_{8}, e_{8} \oplus e_{8} \oplus e_{8}$ as well as the Monster theory. In section 4 we then apply this technique to the proposed self-dual meromorphic conformal field theories at $c=24 k$, and find that the corresponding null vector seems to arise at levels that are too low (for $k \geq 42$ ).

\section{The modular differential equation}

Let us begin by reviewing the definition of the $C_{2}$ criterion of Zhu [10]. We denote the vacuum representation of the chiral algebra by $\mathcal{H}_{0}$, and the fields of the chiral algebra that generate $\mathcal{H}_{0}$ from the vacuum state $\Omega$ by $S^{i}$; the conformal weight of $S^{i}$ is $h_{i}$, where $h_{i}$ is a positive integer. In the following we shall only consider bosonic bosonic conformal field theories, although the main ideas will also apply to fermionic theories. We shall also assume that the spectrum of $L_{0}$ is bounded from below by zero, and that there is a unique vector with $L_{0} \Omega=0$, the vacuum $\Omega$.

With these preparations we now define the subspace $O_{[2]}$ of $\mathcal{H}_{0}$ (we are using the same notation as in [11]) as the vector space that is spanned by the vectors of the form

$$
S_{-h_{i}-1}^{i} \phi, \quad \text { where } \phi \in \mathcal{H}_{0} \text {. }
$$

A chiral algebra (or vertex operator algebra) satisfies the $C_{2}$-criterion, if $O_{[2]}$ has finite codimension in $\mathcal{H}_{0}$, i.e. if the quotient space $A_{[2]}=\mathcal{H}_{0} / O_{[2]}$ is finite-dimensional. It was conjectured by Zhu [10] that all rational conformal field theories satisfy the $C_{2}$ criterion. This has also been confirmed in numerous cases. Obviously, if the chiral algebra satisfies the $C_{2}$-criterion, there exists a positive integer $s_{0}$ such that

$$
L_{-2}^{s_{0}} \Omega \in O_{[2]} .
$$

For the discussion of the characters (or torus amplitudes) a different, but closely related quotient space is of relevance. To define it, we consider the ring of modular forms $\mathbb{C}\left[E_{4}(q), E_{6}(q)\right]$ that is generated by the Eisenstein series $E_{4}(q)$ and $E_{6}(q)$. Recall that a modular form of weight $k$ is a function $f(\tau)$ satisfying

$$
f\left(\frac{a \tau+b}{c \tau+d}\right)=(c \tau+d)^{k} f(\tau),
$$

where as always $q=e^{2 \pi i \tau}$. We furthermore require that $f(\tau)$ has a Taylor series expansion in non-negative integer powers of $q$. The Eisenstein series $E_{4}$ and $E_{6}$ are modular forms 
of weight 4 and 6 , respectively, and they freely generate the ring of all modular forms. Our conventions for the Eisenstein series are

$$
\begin{aligned}
& E_{2}(q)=1-24 q-72 q^{2}-96 q^{3}-168 q^{4}-144 q^{5}-288 q^{6}-\cdots \\
& E_{4}(q)=1+240 q+2160 q^{2}+6720 q^{3}+17520 q^{4}+30240 q^{5}+60480 q^{6}+\cdots, \\
& E_{6}(q)=1-504 q-16632 q^{2}-122976 q^{3}-532728 q^{4}-1575504 q^{5}-4058208 q^{6}-\cdots
\end{aligned}
$$

The Eisenstein series $E_{2}(q)$ is not a modular form since it has a conformal anomaly

$$
E_{2}\left(\frac{a \tau+b}{c \tau+d}\right)=(c \tau+d)^{2}\left(E_{2}(\tau)+\frac{6}{i \pi} \frac{c}{c \tau+d}\right)
$$

It will also play an important role in the following.

With these preparations we now consider the 'module' $\mathcal{H}_{0}\left[E_{4}(q), E_{6}(q)\right]$ of the vacuum representation $\mathcal{H}_{0}$ over $\mathbb{C}\left[E_{4}(q), E_{6}(q)\right]$; this consists of linear combinations of vectors in $\mathcal{H}_{0}$, where the coefficients are polynomials in $E_{4}(q)$ and $E_{6}(q)$. We then define the subspace $O_{q}\left(\mathcal{H}_{0}\right)$ of $\mathcal{H}_{0}\left[E_{4}(q), E_{6}(q)\right]$ to be generated by the vectors of the form

$$
S_{\left[-h_{1}-1\right]}^{i} \phi+2 \sum_{k=2}^{\infty}(2 k-1) \zeta(2 k) E_{2 k}(q) S_{\left[2 k-h_{i}-1\right]}^{i} \phi \quad \phi \in \mathcal{H}_{0} .
$$

Here $E_{2 k}$ for $k \geq 4$ are the higher Eisenstein series that can be written in terms of polynomials in $E_{4}$ and $E_{6}$. The modes $S_{[n]}$ are the natural modes on the torus and can be expressed in terms of the original modes as (see [10, (4.2.3)] for an explicit formula)

$$
S_{[n]}^{i}=S_{n}^{i}+\sum_{m \geq 1} c_{m, n}\left(h_{i}\right) S_{n+m}^{i},
$$

where $c_{m, n}\left(h^{i}\right)$ are constants.

The motivation for the definition of $O_{q}\left(\mathcal{H}_{0}\right)$ comes from the fact that if $\psi \in O_{q}\left(\mathcal{H}_{0}\right)$ then

$$
\operatorname{Tr}_{\mathcal{H}_{j}}\left(V_{0}(\psi) q^{L_{0}-\frac{c}{24}}\right)=0
$$

where $\mathcal{H}_{j}$ is an arbitrary representation of the chiral conformal field theory. Put differently, $O_{q}\left(\mathcal{H}_{0}\right)$ describes the subspace of $\mathcal{H}_{0}\left[E_{4}(q), E_{6}(q)\right]$ whose one-point torus amplitudes vanish (see also [12]).

It is obvious from the above definitions that one can think of $O_{q}\left(\mathcal{H}_{0}\right)$ as a 'deformation' of $O_{[2]}$. It is then easy to see (and explained in [10]) that if a chiral conformal field theory satisfies the $C_{2}$ criterion, then there exists a positive integer $s$, such that

$$
\left(L_{[-2]}^{s}+\sum_{r=0}^{s-1} f_{r}(q) L_{[-2]}^{r}\right) \Omega \in O_{q}\left(\mathcal{H}_{0}\right)
$$

where each $f_{r}(q)$ is polynomial in $E_{4}$ and $E_{6}$.

The smallest such integer $s$ will be called the size of the chiral algebra $\mathcal{A}$. Because of the grading of $\mathcal{H}_{0}\left[E_{4}(q), E_{6}(q)\right]$ (in terms of modular weight and conformal weight with 
respect to $\left.L_{[0]}\right)$ one can show that (2.8) implies that (2.2) holds for the same $s^{*}$, i.e. that $L_{-2}^{s} \Omega \in O_{[2]}$. The converse may in general not be true, although I do not know of any explicit counterexample.

If we insert the zero mode of the vector (2.8) into the character of an arbitrary representation we find that it vanishes, given the definition of $O_{q}\left(\mathcal{H}_{0}\right)$. On the other hand, using standard conformal field theory techniques, Zhu showed [10] that inside the trace, the zero mode of (2.8) can be expressed in terms of polynomials of $L_{0}$, involving as coefficients polynomials in the Eisenstein series $E_{2}, E_{4}$ and $E_{6}$. In turn, each $L_{0}$ can be expressed in terms of a derivative with respect to $q$, and one thus obtains a differential equation of the form

$$
\left[\left(q \frac{d}{d q}\right)^{s}+\sum_{0 \leq r<s} \hat{f}_{r}(q)\left(q \frac{d}{d q}\right)^{r}\right] \chi_{j}(q)=0
$$

where the $\hat{f}_{r}(q)$ are polynomials in the Eisenstein series $E_{2}, E_{4}$ and $E_{6}$ (that are independent of which character $\chi_{j}$ is being considered). This equation can be thought of as being the modular differential equation of [8, 9] (for earlier work see [15, 16]; further developments are described in [17, 18, 19]). Using the fact that it has to transform covariantly under the modular group, it can be brought into the form

$$
\left[D^{s}+\sum_{r=0}^{s-2} f_{r}(q) D^{r}\right] \chi_{j}(q)=0
$$

where each $f_{r}(q)$ is a polynomial in $E_{4}(q)$ and $E_{6}(q)$ of modular weight $2(s-r)$, and

$$
D^{r}=\operatorname{cod}_{(2 r-2)} \cdots \operatorname{cod}_{(2)} \operatorname{cod}(0) \cdot
$$

Here $\operatorname{cod}_{s}$ is the modular covariant derivative that maps a modular form of weight $s$ to one of weight $s+2$,

$$
\operatorname{cod}_{(s)}=q \frac{d}{d q}-\frac{s}{12} E_{2}(q)
$$

Note that the modular anomaly of $E_{2}(q)$ is crucial in order for this to be modular covariant.

\section{The order of the differential equation}

It is believed [8, 9] that the minimal order of the differential equation always agrees with the number of independent characters, i.e. with the number of irreducible representations of the chiral algebra, where pairs of conjugate representations (that lead to the same character) are only counted once. While this is true in many cases (that were checked in [8, 9]), it cannot be true for self-dual conformal field theories. (Self-dual chiral algebras

\footnotetext{
*This will be explained in [13] where a more comprehensive description of the whole approach will be given.

${ }^{\dagger}$ It would be very interesting to understand the relation between this differential equation and the one recently considered in [14].
} 
are characterised by the property that they only possess one representation, namely the vacuum representation itself.) Indeed, if this was so, the minimal order of the modular differential equation for self-dual chiral algebras would have to be one; but then it would necessarily have to be of the form

$$
q \frac{d}{d q} \chi=0
$$

which only has the trivial solution, $\chi=1$. It therefore follows that the minimal order of the modular differential equation cannot always agree with the number of independent characters. In fact, the present argument shows that the minimal order of the modular differential equation is always at least two.

In the following we want to argue that instead the minimal order of the modular differential equation always agrees with the size of the chiral algebra, i.e. with the smallest $s$ for which (2.8) holds. This circumvents the above problem since (2.8) implies that $L_{-2}^{s} \Omega \in O_{[2]}$, which is only possible for $s \geq 2$. To see this we recall that the non-trivial fields of the theory all have $h_{i} \geq 1$. It is therefore impossible to find a null-vector relation that would make $L_{-2} \Omega$ an element of $O_{[2]}$, and hence $s=1$ in (2.2) is never possible.

One direction of this proposed correspondence is straightforwardly proven: the argument of the previous section implies that we can always find a modular differential equation at order $s$ if $s$ is the size of the chiral algebra. The above statement therefore amounts to the assertion that also the converse is true, i.e. if all characters of the conformal field theory satisfy (2.10) for some $s$, that there exists a relation of the form (2.8) with the same $s$. This should follow from the arguments of Zhu [10] - see the more comprehensive description in [13].

For the moment we shall not attempt to prove this, but rather give examples that suggest the truth of this assertion. In each case we shall show that the order of the differential equation is equal to the smallest $s_{0}$ for which (2.2) holds. In particular, this then shows that if the theory satisfies a modular differential equation of order $s$, then $L_{-2}^{s} \Omega \in O_{[2]}$, which is the main conjecture relevant for the analysis of the extremal conformal field theories at $c=24 k$. We begin with some simple cases for which the analysis can be done completely.

\subsection{Minimal models}

The Virasoro minimal models arise for the central charges $c=c_{p, q}$ with

$$
c_{p, q}=1-\frac{6(p-q)^{2}}{p q},
$$

where $p, q \geq 2$ are coprime integers. They define rational conformal field theories with $(p-1)(q-1) / 2$ inequivalent highest weight representations; the corresponding conformal weights are given by

$$
h_{(r, s)}=\frac{(r p-q s)^{2}-(p-q)^{2}}{4 p q},
$$

where $1 \leq r \leq q-1$ and $1 \leq s \leq p-1$, and we have the identification $h_{r, s}=h_{q-r, p-s}$. For Virasoro minimal models the analysis of [8, 9] applies, and it follows that the order of the 
modular differential equation is precisely equal to the number of independent characters, i.e. to $(p-1)(q-1) / 2$.

For Virasoro theories the space $A_{[2]}$ can be taken to be generated by the vectors $L_{-2}^{l} \Omega$, where $l=0,1, \ldots$ For $c=c_{p, q}$ the vacuum Verma module has a null-vector at level $(p-1)(q-1)$ for which the coefficient of $L_{-2}^{(p-1)(q-1) / 2} \Omega$ does not vanish [20]. This implies that $L_{-2}^{(p-1)(q-1) / 2} \Omega$ is in fact in $O_{[2]}$ (since it differs from vectors in $O_{[2]}$ by a null-vector), and thus that the minimal $s_{0}$ in $(2.2)$ is indeed $(p-1)(q-1) / 2$.

\section{2 $\mathrm{SU}(2) \mathrm{WZW}$ models at level $k$}

The next simple class of models for which we can give a complete description are the $\mathrm{su}(2)$ current theories at level $k$. It is well known that these chiral algebras have $k+1$ irreducible inequivalent representations, namely those characterised by the spin $j$ of the highest weight space with $j=0, \frac{1}{2}, \ldots, \frac{k}{2}$. Again the analysis of [8, 9] applies, and it follows that the order of the modular differential equation is $k+1$. This was also worked out explicitly in [8] for the case $k=1$.

On the other hand, the quotient space $A_{[2]}$ for these models was analysed in [21]. For $k=1$ we found

$$
A_{[2]}\left(\widehat{s u}(2)_{1}\right)=\mathbf{1}_{0} \oplus \mathbf{3}_{1} \oplus \mathbf{1}_{2}
$$

where we have decomposed $A_{[2]}$ in terms of representations of the zero modes of $\widehat{s u}(2)$, and the index indicates at which conformal weight theses states appear. [So for example, $\mathbf{3}_{1}$ refers to the three states $J_{-1}^{a} \Omega$, etc.] The state at level two is precisely $L_{-2} \Omega$, which is therefore not in $O_{[2]}$. On the other $L_{-2}^{2} \Omega=0$ in $A_{[2]}$, and hence the minimal $s_{0}$ in (2.2) is $s_{0}=2=k+1$, in agreement with the above.

For $k=2$ we find instead

$$
A_{[2]}\left(\widehat{s u}(2)_{2}\right)=\mathbf{1}_{0} \oplus \mathbf{3}_{1} \oplus \mathbf{1}_{2} \oplus \mathbf{5}_{2} \oplus \mathbf{3}_{3} \oplus \mathbf{1}_{4}
$$

The states $\mathbf{1}_{2}$ and $\mathbf{1}_{4}$ correspond to $L_{-2} \Omega$ and $L_{-2}^{2} \Omega$, respectively, and thus only the state $L_{-2}^{3} \Omega \in O_{[2]}$. Thus the minimal $s_{0}$ in (2.2) is $s_{0}=3=k+1$, as expected. For $k=3$ we find

$$
A_{[2]}\left(\widehat{s u}(2)_{3}\right)=\mathbf{1}_{0} \oplus \mathbf{3}_{1} \oplus \mathbf{1}_{2} \oplus \mathbf{5}_{2} \oplus \mathbf{3}_{3} \oplus \mathbf{7}_{3} \oplus \mathbf{1}_{4} \oplus \mathbf{5}_{4} \oplus \mathbf{3}_{5} \oplus \mathbf{1}_{6}
$$

Now the singlet vectors correspond to $L_{-2}^{l} \Omega$ with $l=0,1,2,3$, and hence only $L_{-2}^{4} \Omega \in O_{[2]}$, leading to the minimal $s_{0}=4=k+1$, again as expected.

It is not difficult to guess now how the structure will continue for all $k$ : at every $k$, $A_{[2]}\left(\widehat{s u}(2)_{k}\right)$ will contain the singlet vectors $\mathbf{1}_{2 n}$ with $n=0,1,2, \ldots, k$, corresponding to $L_{-2}^{n} \Omega$. Thus only $L_{-2}^{k+1} \Omega \in O_{[2]}$, leading to the minimal $s_{0}=k+1$.

\section{3 $\mathrm{SU}(3) \mathrm{WZW}$ model at level $k=1,2$}

Unfortunately, the analysis of the quotient space $A_{[2]}$ becomes increasingly complicated for affine algebras of higher rank, and we do not know any general formulae beyond $\operatorname{su}(2)$. However, we can still give the results for low levels, for example for $\operatorname{su}(3)$ at $k=1$ and $k=2$. 
For $k=1$ there are three irreducible representations (namely the vacuum representation, as well as those associated to $\mathbf{3}$ and $\overline{\mathbf{3}}$ of su(3)); since the $\mathbf{3}$ and $\overline{\mathbf{3}}$ representations are conjugate representations, they lead to the same character and we thus expect to find a second order modular differential equation. On the other hand, the $A_{[2]}$ space consists of [21]

$$
A_{[2]}\left(\widehat{s u}(3)_{1}\right)=\mathbf{1}_{0} \oplus \mathbf{8}_{1} \oplus \mathbf{1}_{2} \oplus \mathbf{8}_{2} \oplus \mathbf{1}_{3},
$$

and thus $L_{-2}^{l} \Omega$ with $l=0,1$ is in $A_{[2]}$ - these are the two singlet states at levels 0,2 but $L_{-2}^{2} \Omega \in O_{[2]}$, showing that $\operatorname{su}(3)$ at level $k=1$ has indeed minimal $s_{0}=2$.

At level $k=2$, there are six irreducible representations — in addition now also the $\mathbf{6}$, $\overline{\mathbf{6}}$ and $\mathbf{8}$ appear — but two of them are complex conjugates of one another, and hence we expect a fourth order differential equation. On the other hand, the $A_{[2]}$ space consists of 21 .

$$
\begin{aligned}
A_{[2]}\left(\widehat{s u}(3)_{2}\right)= & \mathbf{1}_{0} \oplus \mathbf{8}_{1} \oplus \mathbf{1}_{2} \oplus \mathbf{8}_{2} \oplus \mathbf{2}_{2} \oplus \mathbf{1}_{3} \oplus \mathbf{1 0}_{3} \oplus \overline{\mathbf{1 0}}_{3} \oplus \mathbf{8}_{3} \oplus \mathbf{2}_{3} \\
& \oplus \mathbf{1}_{4} \oplus 2 \cdot \mathbf{8}_{4} \oplus \mathbf{2} \mathbf{7}_{4} \oplus \mathbf{1}_{5} \oplus \mathbf{8}_{5} \oplus \mathbf{1}_{6} .
\end{aligned}
$$

This is in agreement with the expectation that $L_{-2}^{4} \Omega \in O_{[2]}$, but that no smaller power of $L_{-2}$ satisfies this condition.

So far we have discussed rational conformal field theories for which the order of the modular differential equation coincides precisely with the number of independent characters, as suggested in [8, 9]. Incidentally, these are also the theories for which Zhu's algebra $A_{[1,1]}$ [10] has the same dimension as $A_{[2]}$ [21], see also [22, 23] . However, one would expect potential problems with our proposal to arise for those cases where $\operatorname{dim} A_{[1,1]}<\operatorname{dim} A_{[2]}$. In particular, this happens for the self-dual theories since they have $\operatorname{dim} A_{[1,1]}=1$, whereas $\operatorname{dim} A_{[2]} \geq 2$ because $A_{[2]}$ always contains at least the vacuum as well as $L_{-2} \Omega$.

\subsection{The self-dual $e_{8}$ level 1 theory.}

The simplest self-dual conformal field theory (and conjecturally the only self-dual conformal field theory at $c=8-$ see [2, 24]) is the $e_{8}$ affine theory at $k=1$. The vacuum character of this theory is simply

$\chi_{e_{8}}(q)=j(q)^{1 / 3}=q^{-1 / 3}\left(1+248 q+4124 q^{2}+34752 q^{3}+213126 q^{4}+1057504 q^{5}+\cdots\right)$.

Since it is the only character of this chiral algebra, we can systematically search for the differential equation of the type (2.10) of smallest order that annihilates $\chi_{e_{8}}(q)$. One easily finds that

$$
\left[D^{2}-\frac{1}{6} E_{4}(q)\right] \chi_{e_{8}}(q)=0 .
$$

On the other hand, the quotient space $A_{[2]}=\mathcal{H}_{0} / O_{[2]}$ for this theory was determined explicitly in [21], where it was found to consist of the $e_{8}$ representations

$$
A_{[2]}=\mathbf{1}_{0} \oplus \mathbf{2 4 8}_{1} \oplus \mathbf{3 8 7 5} \mathbf{5}_{2} \oplus \mathbf{1}_{2}
$$

\footnotetext{
$\ddagger$ The question of when these dimensions agree or disagree was in fact the motivation for determining the structure of $A_{[2]}$ for the various examples in [21. An introduction to Zhu's algebra can be found in [22, 23].
} 
and the index denotes again the conformal weight at which these states appear. To obtain this result we used that the complete null-space of the $e_{8}$ level $k=1$ theory is generated from the null vectors that lie in the $\mathbf{2 7 0 0 0}$ representation of $e_{8}$ at conformal weight $h=2$. Furthermore, we may take $A_{[2]}$ to be generated by the completely symmetric powers of the 'adjoint' generators $J_{-1}^{a}$ acting on the vacuum, since any commutator term will automatically lie in $O_{[2]}$. To determine $A_{[2]}$ we therefore have to determine the intersection of descendants of the states in the $\mathbf{2 7 0 0 0}$ with symmetrised tensor products of $J_{-1}^{a}$ acting on the vacuum. This can be analysed per computer (using LiE and a C-program).

The only singlet at level 2 is the state $L_{-2} \Omega$, and since it survives in $A_{[2]}$, it is not in $O_{[2]}$; on the other hand, $A_{[2]}$ does not contain any states at level 4 , and thus $L_{-2}^{2} \Omega \in O_{[2]}$. Thus it follows that the $e_{8}$ level $k=1$ theory has indeed minimal $s_{0}=2$, in agreement with the order of the modular differential equation (3.10).

In contrast to the situation discussed in [8, 9], the modular differential equation has higher order (namely two) than the number of independent characters (which is one here). One may therefore ask what the other solutions of the modular differential equation correspond to. For the case at hand, there is only one additional solution (in addition to $\left.\chi_{e_{8}}(q)\right)$, which is of the form

$$
\hat{\chi}(q)=q^{\frac{1}{2}}\left(1+\frac{228}{11} q+\frac{34938}{187} q^{2}+\frac{5163352}{4301} q^{3}+\cdots\right) .
$$

This does not seem to correspond to a character of any representation; in particular, the coefficients in the $q$-expansion do not seem to be integers $\$$ I suspect that the $e_{8}$ level $k=1$ theory has a second (independent) modular differential equation at third order (which comes from the fact that also $L_{-2}^{3} \Omega \in O_{[2]}$ ), and that only $\chi_{e_{8}}$ (but not $\hat{\chi}(q)$ ) is a solution to both differential equations. (It is not difficult to find such a third order modular differential equation, but it is not uniquely determined by this constraint.) I suspect that the same will also happen for the other self-dual examples that we are about to discuss.

\subsection{The self-dual $e_{8} \oplus e_{8}$ theory}

The next simplest self-dual conformal field theory is the tensor product of two such theories at $c=16$. The corresponding character is

$$
\chi_{e_{8} \oplus e_{8}}(q)=j(q)^{2 / 3}=q^{-2 / 3}\left(1+496 q+69752 q^{2}+2115008 q^{3}+34670620 q^{4}+\cdots\right) .
$$

Again, we can systematically search for the differential equation of the type (2.10) of smallest order that annihilates $\chi_{e_{8} \oplus e_{8}}(q)$, and we find that

$$
\left[D^{3}+\frac{5}{9} E_{6}(q)\right] \chi_{e_{8} \oplus e_{8}}(q)=0 .
$$

\footnotetext{
${ }^{\S}$ I have determined them up to order $q^{11}$, and the denominator continues to contain new prime-factors as one increases the order of the expansion.

The two coefficients in front of the two terms $E_{6}$ and $E_{4} D$ are not uniquely determined by this condition; we have given the result for the case that the $E_{4} D$ term is absent.
} 
Thus we expect that this chiral algebra has minimal $s_{0}=3$. If we denote the Virasoro generators of the two $e_{8}$ copies by $L^{(1)}$ and $L^{(2)}$, then we know from the previous analysis that $L_{-2}^{(j)} \Omega \notin O_{[2]}$, but $L_{-2}^{(j) 2} \Omega \in O_{[2]}$. Since $L=L^{(1)}+L^{(2)}$, we find that

$$
\begin{aligned}
& L_{-2} \Omega=L_{-2}^{(1)} \Omega+L_{-2}^{(2)} \Omega \notin O_{[2]} \\
& L_{-2}^{2} \Omega=L_{-2}^{(1) 2} \Omega+L_{-2}^{(2) 2} \Omega+2 L_{-2}^{(1)} L_{-2}^{(2)} \Omega \notin O_{[2]} \\
& L_{-2}^{3} \Omega=L_{-2}^{(1) 3} \Omega+3 L_{-2}^{(1) 2} L_{-2}^{(2)} \Omega+3 L_{-2}^{(1)} L_{-2}^{(2) 2} \Omega+L_{-2}^{(2) 3} \Omega \in O_{[2]}
\end{aligned}
$$

in agreement with the above expectation. Note that the last term in (3.15) is not in $O_{[2]}$ since $L_{-2}^{(1)} L_{-2}^{(2)} \Omega \notin O_{[2]}$, but that the expression in the third line is in $O_{[2]}$ since $L_{-2}^{(j) 2} \Omega \in O_{[2]}$. We are using here that $O_{[2]}\left(\mathcal{H}_{1} \oplus \mathcal{H}_{2}\right)=O_{[2]}\left(\mathcal{H}_{1}\right) \oplus O_{[2]}\left(\mathcal{H}_{2}\right)$, as is obvious from the definition of the $O_{[2]}$ space.

\subsection{The Monster theory}

The most interesting self-dual conformal field theory is probably the Monster conformal field theory constructed by Frenkel, Lepowsky and Meurman [3] (see also [4]). Its partition function is the well known $J(q)=j(q)-744$ function

$$
\chi_{M}=q^{-1}\left(1+196884 q+21493760 q^{2}+864299970 q^{3}+20245856256 q^{4}+\cdots\right) .
$$

One again finds that it satisfies a third order modular differential equation which is now uniquely determined by this constraint to be

$$
\left[D^{3}+\frac{16}{31} E_{6}(q)-\frac{290}{279} E_{4}(q) D\right] \chi_{M}(q)=0 .
$$

Thus we again expect the Monster theory to have minimal $s_{0}=3$. This can also be independently confirmed. As we mentioned before, for any non-trivial theory $L_{-2} \Omega \notin O_{[2]}$. Since the Monster theory does not contain any fields with $h=1, O_{[2]}$ does not contain any states at level 4 since any such state would have to be of the form $S_{-3} \phi$ for some $S$ of $h=2$, and with $\phi$ at level one. But since the Monster theory does not contain any states at level one, no such element of $O_{[2]}$ can exist. At level six, on the other hand, this argument breaks down. At level six we have singlet states with respect to the Monster group that arise from Virasoro descendants of the vacuum, namely

$$
L_{-2}^{3} \Omega, \quad L_{-3}^{2} \Omega, \quad L_{-4} L_{-2} \Omega, \quad L_{-6} \Omega .
$$

In addition we can get three singlet states of the form (see also [28, 29])

$$
\sum_{i j} c_{i j} L_{-2} W_{-2}^{i} W_{-2}^{j} \Omega, \quad \sum_{i j} c_{i j} W_{-3}^{i} W_{-3}^{j} \Omega, \quad \sum_{i j} c_{i j} W_{-4}^{i} W_{-2}^{j} \Omega
$$

where the $W^{i}$ denote the 196883 fields of conformal weight $h=2$ that transform in an irreducible Monster representation, and the $c_{i j}$ are the coefficients that pick out the trivial Monster representation in this tensor product. Finally, we have the singlet state

$$
\sum_{\alpha \beta} d_{\alpha \beta} \hat{W}_{-3}^{\alpha} \hat{W}_{-3}^{\beta} \Omega
$$


where the $\hat{W}^{\alpha}$ denote the 21296876 Virasoro primary fields of conformal weight $h=3$ that also transform in an irreducible Monster representation; the constants $d_{\alpha \beta}$ are again the appropriate Clebsch-Gordon coefficients.

Of these eight states, only the first states in (3.18) and (3.19), and the state in (3.20) do not lie manifestly in $O_{[2]}$. Now it is known from the decomposition of coefficients in (3.16) (see for example [25]) that at level 6, there are only as many Monster invariant states as there are Virasoro descendant:I, namely four. Thus we must have four linear relations between these eight states. Unless there are some unexpected cancellations, we therefore expect that these relations allow us to rewrite $L_{-2}^{3} \Omega$ in terms of elements in $O_{[2]}$. Thus we expect that $L_{-2}^{3} \Omega \in O_{[2]}$, i.e. that the minimal $s_{0}$ in (2.2) is $s_{0}=3$ for the Monster theory.

\subsection{The other self-dual theories at $c=24$}

At $c=24$ there are a number of other self-dual theories; these include, in particular, the lattice theories and their orbifolds [30, 31]. (A complete list has been conjectured in [7].)

Their characters differ from that of the Monster by a constant $K, \chi(q)=J(q)+K$. Provided that $K \neq 744, \chi(q)$ also satisfies a third order differential equation of the same type as (3.17). Unfortunately, we cannot directly calculate the $A_{[2]}$ spaces for these examples, and we cannot therefore compare this to the minimal value of $s_{0}$ in (2.2). However, the case $K=744$ is interesting, since this describes precisely the character that occurs for the $e_{8} \oplus e_{8} \oplus e_{8}$ theory,

$$
\chi_{e_{8} \oplus e_{8} \oplus e_{8}}(q)=J(q)+744=j(q) .
$$

In this case, $\chi_{e_{8} \oplus e_{8} \oplus e_{8}}(q)$ does not satisfy a third order equation, but only a fourth order equation. This is in perfect agreement with the fact that only $L_{-2}^{4} \Omega \in O_{[2]}$ since

$$
L_{-2}^{3} \Omega=6 L_{-2}^{(1)} L_{-2}^{(2)} L_{-2}^{(3)} \Omega+v \notin O_{[2]}, \quad v \in O_{[2]},
$$

where $L_{n}^{(i)}, i=1,2,3$ are the Virasoro modes of the $i^{\text {th }} e_{8}$ theory. The same argument implies also that the theory $e_{8}^{\oplus l}$ has minimal $s_{0}=l+1$. At least for the first few $l$ this agrees with the order of the corresponding modular differential equation **

\section{Extremal self-dual CFTs at $c=24 k$}

We now want to apply these ideas to the extremal self-dual conformal field theories at $c=24 k$ that were recently proposed by Witten [1]. As is explained there, the condition that the theories are extremal and self-dual determines their partition function uniquely. The assumption of extremality means that the vacuum character is of the form

$$
\chi_{k}=q^{-k}\left(\prod_{n=2}^{\infty} \frac{1}{1-q^{n}}+\mathcal{O}\left(q^{k+1}\right)\right) .
$$

\footnotetext{
$\|$ This was already noted in [26, 24, 27].

${ }^{* *}$ I thank the referee for drawing my attention to this point.
} 
In terms of the representation space $\mathcal{H}_{0}$ this means that, up to level $k$, it is generated from the vacuum by the Virasoro modes. Thus the fields of the chiral algebra contain, apart from the stress energy tensor with $h=2$, only primary fields with $h_{i} \geq k+1$.

As before for the examples in section $3.4-3.6$ we can now determine the lowest order modular differential equation that annihilates $\chi_{k}$. Since the modular differential equation is covariant with respect to the modular group, we are looking for an equation of the form

$$
\left[D^{s}+\sum_{r=0}^{s-2} f_{r}(q) D^{r}\right] \chi_{k}(q)=0 .
$$

We want to determine the minimal value of $s$ for which such an equation exists. Let us denote by $h(s)$ the number of monomials in $E_{4}$ and $E_{6}$ that have total modular weight $2 s$. The total number of free parameters in a modular differential equation of order $s$ is then

$$
p(s)=\sum_{t=2}^{s} h(t)
$$

since each $f_{r}(q)$ in (4.2) is a modular form of weight $2(s-r)$. The first few values of $p(s)$ (together with the corresponding values of $h(s)$ ) are tabulated in table 1 . It is easy to see that asymptotically $h(s) \sim C_{1} s$ and $p(s) \sim C_{2} s^{2}$.

\begin{tabular}{|c|c|c||c|c|c||c|c|c||c|c|c|}
\hline$s$ & $h(s)$ & $p(s)$ & $s$ & $h(s)$ & $p(s)$ & $s$ & $h(s)$ & $p(s)$ & $s$ & $h(s)$ & $p(s)$ \\
\hline 2 & 1 & 1 & 8 & 2 & 9 & 14 & 3 & 23 & 20 & 4 & 43 \\
\hline 3 & 1 & 2 & 9 & 2 & 11 & 15 & 3 & 26 & 21 & 4 & 47 \\
\hline 4 & 1 & 3 & 10 & 2 & 13 & 16 & 3 & 29 & 22 & 4 & 51 \\
\hline 5 & 1 & 4 & 11 & 2 & 15 & 17 & 3 & 32 & 23 & 4 & 55 \\
\hline 6 & 2 & 6 & 12 & 3 & 18 & 18 & 4 & 36 & 24 & 5 & 60 \\
\hline 7 & 1 & 7 & 13 & 2 & 20 & 19 & 3 & 39 & 25 & 4 & 64 \\
\hline
\end{tabular}

Table 1: Number of monomials $h(s)$ of $E_{4}$ and $E_{6}$ of modular weight $2 s$, and of free parameters $p(s)$ for a modular differential equation of order $s$.

Now it is easy to see that if the equation of the form (4.2) annihilates the first $k+h(s)$ powers of $q$ (starting from $q^{-k}$ up to $q^{h(s)-1}$ ), then the equation will hold identically, i.e. for each power $q^{n}$. The reason for this is simple: if the differential equation annihilates the negative powers of $q$, then the resulting function is a power series in $q$ of modular weight $2 s$, and hence must be a polynomial in $E_{4}$ and $E_{6}$ (of appropriate degree). It is then uniquely characterised by the first $h(s)$ coefficients, starting from $q^{0}$ to $q^{h(s)-1}$. If all of these coefficients vanish, the function itself therefore has to vanish identically.

Thus we need to choose the order of differential equation such that the number of free parameters, $p(s)$ is at least as big as $k+h(s)$, i.e.

$$
p(s) \geq k+h(s) \Longleftrightarrow k \leq p(s-1)
$$


Now consider $k=42$ and $s=21$. According to what we said above we need to fix $k+h(s)=42+4=46$ constants, which is less then $p(s)=47$. Thus we expect to have an order $s=21$ modular differential equation annihilating $\chi_{42}$, and we have checked explicitly, using Maple, that this is indeed the case $\dagger^{\dagger}$ Such a differential equation should imply that

$$
\Phi=L_{-2}^{21} \Omega \in O_{[2]} .
$$

On the other hand, $\Phi$ appears at level 42 above the vacuum, but for $k=42$ the proposed conformal field theory only contains Virasoro descendants at this level. (The additional generators of the proposed conformal field theory only appear at levels greater or equal than $k+1=43$.) Since $c>1$ there is no null-vector relation between these Virasoro descendants, and thus (4.5) is impossible. Thus either our claim regarding the correspondence between the modular differential equation and the structure of $A_{[2]}$ breaks down at this stage, or there is a contradiction.

Since $p(s)$ grows quadratically, it is clear that for all values of $k \geq 42$ we obtain such a problem. (Strictly speaking, we are assuming here, that the partition function is sufficiently generic so that we can actually find a modular differential equation of this order. Given that we could find such a differential equation for $k=42$, it seems very plausible that this will also be the case for $k \geq 43$.) For the first few $k \geq 42$ the order of the minimal modular differential equation is summarised in table 2.

\begin{tabular}{|c|c||c|c||c|c||c|c|}
\hline$k$ & $s$ & $k$ & $s$ & $k$ & $s$ & $k$ & $s$ \\
\hline 42 & 21 & 44 & 22 & 46 & 22 & 48 & 23 \\
\hline 43 & 21 & 45 & 22 & 47 & 22 & 49 & 23 \\
\hline
\end{tabular}

Table 2: The minimal order $s$ of the differential equation for the extremal self-dual theories at $c=24 k$.

This analysis therefore suggests that at least the theories with $k \geq 42$ are inconsistent. On the other hand, by considering higher genus amplitudes, [1, 6] found impressive evidence that the theories with $k=2$ and $k=3$ may indeed be consistent. The explicit genus two analysis of [6] can also be generalised to $k \geq 3$, but it stops being a real consistency check at $k=11$, and thus their result is not in any contradiction with the above suggestions.

If these conclusions are correct, it would probably mean that one has to adjust the chiral conformal field theory of the $A d S_{3}$ description, and add some (few) states at lower conformal weight. The conformal weight at which these additional states appear is of order $\sqrt{k}$ for large $k$; their conformal dimension therefore goes to infinity in the large $k$ limit, and the existence of these states does not lead to a contradiction with the semiclassical

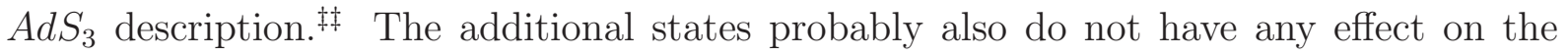
leading order entropy calculation of [1]. It would be very interesting to find a 'minimal' proposal for a consistent chiral conformal field theory satisfying these constraints.

\footnotetext{
${ }^{\dagger \dagger}$ I thank Marco Baumgartl for helping me do this calculation.

${ }_{\ddagger \ddagger}$ I thank Edward Witten for pointing this out to me.
} 
That something of this nature is necessary is maybe not too surprising in view of the superconformal analysis of [1]: there it was shown that the consistency of the R-sector requires that one has to add NS-sector states at conformal weight zero (corresponding to $q^{0}$ ) at least for $k^{*} \geq 8$ (with $k^{*}$ even). If the above analysis is correct, it would seem that something similar (albeit slightly more dramatic) is required in order to make the bosonic conformal field theories consistent.

\section{Acknowledgements}

This research has been partially supported by the Swiss National Science Foundation and the Marie Curie network 'Constituents, Fundamental Forces and Symmetries of the Universe' (MRTN-CT-2004-005104). I thank Marco Baumgartl for help with the Maple calculations, and Ilka Brunner, Terry Gannon, Peter Goddard, Sergei Gukov, Gerald Höhn, Christoph Keller, Ingo Kirsch, Andy Neitzke and Edward Witten for useful correspondences and discussions.

\section{References}

[1] E. Witten, Three-dimensional gravity revisited, arXiv:0706.3359 [hep-th].

[2] P. Goddard, Meromorphic conformal field theory, in: Infinite dimensional Lie algebras and Lie groups: Proceedings of the CIRM Luminy Conference, 1988 (World Scientific, Singapore, 1989) 556.

[3] I. Frenkel, J. Lepowsky and A. Meurman, Vertex operator algebras and the Monster (Academic Press, 1988).

[4] R.E. Borcherds, Vertex operator algebras, Kac-Moody algebras and the Monster, Proc. Natl. Acad. Sci. USA 83 (1986) 3026.

[5] T. Gannon, Moonshine beyond the Monster: The bridge connecting algebra, modular forms and physics, Cambridge University Press (2006).

[6] D. Gaiotto and X. Yin, Genus two partition functions of extremal conformal field theories, arXiv:0707.3437 [hep-th].

[7] A.N. Schellekens, Meromorphic $c=24$ conformal field theories, Commun. Math. Phys. 153 (1993) 159 [arXiv:hep-th/9205072].

[8] S.D. Mathur, S. Mukhi and A. Sen, On the classification of rational conformal field theories, Phys. Lett. B 213 (1988) 303.

[9] S.D. Mathur, S. Mukhi and A. Sen, Reconstruction of conformal field theories from modular geometry on the torus, Nucl. Phys. B 318 (1989) 483.

[10] Y. Zhu, Vertex operator algebras, elliptic functions and modular forms, Caltech preprint (1990), J. Amer. Math. Soc. 9 (1996) 237. 
[11] M.R. Gaberdiel and A. Neitzke, Rationality, quasirationality and finite W-algebras, Commun. Math. Phys. 238 (2003) 305 [arXiv:hep-th/0009235].

[12] E. Witten, Quantum field theory, Grassmannians, and algebraic curves, Commun. Math. Phys. 113 (1988) 529.

[13] M. Gaberdiel and C.A. Keller, in preparation.

[14] P. Bantay and T. Gannon, Conformal characters and the modular representation, JHEP 0602 (2006) 005 [arXiv:hep-th/0512011].

[15] T. Eguchi and H. Ooguri, Conformal and current algebras on general Riemann surface, Nucl. Phys. B 282 (1987) 308.

[16] G. Anderson and G.W. Moore, Rationality in conformal field theory, Commun. Math. Phys. 117 (1988) 441.

[17] W. Eholzer, On the classification of modular fusion algebras, Commun. Math. Phys. 172 (1995) 623 [hep-th/9408160]; Ph.D. thesis (Bonn 1995), hep-th/9502160.

[18] W. Eholzer and N.-P. Skoruppa, Modular invariance and uniqueness of conformal characters, Commun. Math. Phys. 174 (1995) 117 [hep-th/9407074]; Conformal characters and theta series, Lett. Math. Phys. 35 (1995) 197 [hep-th/9410077]

[19] M. Flohr and M.R. Gaberdiel, Logarithmic torus amplitudes, J. Phys. A 39 (2006) 1955 [arXiv:hep-th/0509075].

[20] B.L. Feigin and D.B. Fuchs, Verma modules over the Virasoro algebra, in: Topology, Lecture notes in Mathematics 1060 (Springer, Berlin 1984) 230.

[21] M.R. Gaberdiel and A. Neitzke, unpublished notes (2000).

[22] M.R. Gaberdiel and P. Goddard, Axiomatic conformal field theory, Commun. Math. Phys. 209 (2000) 549 [arXiv:hep-th/9810019].

[23] M.R. Gaberdiel, An introduction to conformal field theory, Rept. Prog. Phys. 63 (2000) 607 [arXiv:hep-th/9910156].

[24] G. Höhn, Selbstduale Vertexoperatorsuperalgebren und das Babymonster, Ph.D. thesis (Bonn 1995), Bonner Mathematische Schriften 286 (1996) [arXiv:0706.0236 [math.QA]].

[25] J. McKay and H. Strauss, The q-series of monstrous moonshine 8 the decomposition of the head characters, Commun. Alg. 18(1) (1990) 253.

[26] C. Dong and G. Mason, Monstrous Moonshine of higher weight, Acta Math. 185 (2000) 101 [arXiv:math/9803116 (math.QA)].

[27] B. Craps, M.R. Gaberdiel and J.A. Harvey, Monstrous branes, Commun. Math. Phys. 234 (2003) 229 [arXiv:hep-th/0202074]. 
[28] A. Matsuo, Norton's trace formulae for the Griess algebra of a vertex operator algebra with larger symmetry, Commun. Math. Phys. 224 (2001) 565.

[29] M. Tuite, The Virasoro algebra and some exceptional Lie and finite groups, SIGMA 3 (2007) 008 [arXiv:math.QA/0610322].

[30] L. Dolan, P. Goddard and P. Montague, Conformal field theory, triality and the Monster group, Phys. Lett. B 236 (1990) 165.

[31] L. Dolan, P. Goddard and P. Montague, Conformal field theories, representations and lattice constructions, Commun. Math. Phys. 179 (1996) 61 [arXiv: hep-th/9410029]. 